\title{
Social and human sciences in health: incorporating the theory
}

When we were planning the edition of a thematic issue focused on theory, we knew that dealing with this topic would not be a simple task. However, as the articles starting coming in, we realized we had ventured into a highly thought-provoking and complex area.

The theme is broad and can lead us in multiple directions; after all, the question of constructing a theoretical concept, as proclaimed in the tradition of ancient Greece, is part of the culture in which "theater," "theory" and philosophy overlap. According to the sociologist Robert Nisbet (1976), it is revealing that the word theoria comes from the same Greek root as the word théatron. Many other words have meanings that encompass these two worlds, which to the Greeks complement each other: theorema can be contemplation, but also spectacle and object of study; theorros is the person who consults the oracle; theorein is to look closely or to speculate.

Presenting the material that addresses the field of social sciences as a theoretical construct in a theatrical evocation made it possible to introduce authors/characters; disciplinary fields/scenarios; theoretical frameworks/plots, not just for contemplation but also for an open spectacle, not as mere speculation, but for a reflective and critical approach.

At the outset, we place the trajectory of the theoretical construct in the sociology of medicine/health discarding the idea that prevailed for some time that it was an atheoretical field and of exclusive application to the medical field. Immediately thereafter, the anthropological analyses begin with a dialogue with medicine/health as a social, cultural and political experience and construct.

The two works - the sociology of health in the United States and in Brazil - seek to demarcate the trajectories of the field in the pioneering US experiences and current theoretical perspectives, and the attempt to create a historical, social and cognitive identity in the case of Brazil.

Undoubtedly, education reveals itself as an ideal platform to reproduce knowledge and train professionals in the area of social sciences. However, as the article shows, discussions between "public sociology," "critical sociology" and "applied sociology" still persist when working at the frontiers of knowledge on the one hand, and when faced with the domination of biomedical knowledge on the other.

Probably, the test is closer to education and the possibilities of instrumentalizing it for health practices rather than for scientific production, where limits, boundaries and borders are continuously crossed in the creation of an interdisciplinary knowledge.

However, in order to achieve this feat, it is important to know how to look for the elements necessary for this knowledge. We opted for five core dimensions to construct a theoretical and methodological body of knowledge: narrative, auto/biography, history and the presence of the individual in health care and ethics. These dimensions complement each other in the health field: all texts, especially in the social sciences and humanities, materialize in the "emplotment" (Hayden White, 1994). All this in a narrative that can be anthropological, sociological, political, historical and enshrined in the dialectics of the autobiographical-report, but at the time of research comes up against the intersubjectivity in the researcher/ researchee relationship.

In the synopses there is a (re)encounter on the one hand with one of the most fertile concepts of anthropology and sociology, namely the gift and its role in human action in health, and on the other with theoretical questioning to comprehend the health practices in the UK.

For the Greeks, the theater had an eminently pedagogical function: to amuse and discuss controversial issues; likewise, by subjugating the theoretical and methodological dimension, the latter ceases to be a simple artifact of research into health and becomes an essential link in the understanding of the reality under scrutiny.

I would like to thank the authors, the scientific editors - Cecília and Romeu - and the executive editorial committee. Without all of you, this issue would not have been possible.

Everardo Duarte Nunes

Guest Editor 